\title{
EVALUACIÓN DE LA PROFUNDIDAD DEL SURCO GINGIVAL DESPUÉS DEL USO DEL HILO RETRACTOR 00 PARA RESTAURACIONES CLASE V EN PACIENTES ATENDIDOS EN LA CLÍNICA ODONTOLÓGICA DE LA UNIVERSIDAD DE LAS AMÉRICAS
}

\author{
EVALUATION OF THE DEPTH OF THE GINGIVAL SURCO AFTER THE USE OF THE \\ RETRACTOR 00 THREAD FOR CLASS $V$ RESTORATIONS IN PATIENTS ATTENDED AT \\ THE DENTAL CLINIC OF THE UNIVERSITY OF THE AMERICAS
}

\begin{abstract}
Sebastián Giacometti Velasco $^{1 \mathrm{a}}$, Alexander Cruz Gallegos $^{2 a}$, Alfredo Quintana Ramírez $^{a}$
RESUMEN

Objetivo: Evaluar la profundidad del surco gingival después del uso del hilo retractor 00 para restauraciones clase $\mathrm{V}$. Materiales y métodos: Se evaluó 84 piezas dentales en 46 pacientes, tanto hombres como mujeres, que asistieron a la Clínica de la Universidad de las Américas. Se realizó el sondaje y control luego de 72 horas para determinar si afectó el hilo retractor a nivel del surco gingival. Resultados: Un $64,29 \%$ de las piezas evaluadas presentaron un cambio, mientras que el $35,71 \%$ se mantuvieron en el estándar normal. En cuanto a la diferenciación de biotipos gingivales se obtuvo un mayor cambio de la medida del surco gingival en pacientes con biotipo delgado, con un $64,81 \%$ de piezas evaluadas en relación con el biotipo grueso, con $35,19 \%$ con base en la medida final. En el presente estudio también se determino la existencia de sangrado a nivel del surco gingival el momento de realizar los sondajes: inicial, medio y final, con resultados en sangrado de 7,69\%, 34,62\% y 57,69\%, respectivamente. Conclusión: Se determinó que existen diferencias significativas con respecto a la profundidad del surco presentada en los sondajes inicial, medio y final; sin embargo, el nivel de profundidad del surco fue superior en el sondaje medio, lo que confirma que el uso del hilo retractor afecta al periodonto al momento de realizar el procedimiento restaurador. En este estudio se demostró que afectó en un $64,29 \%$ de las piezas evaluadas.
\end{abstract}

Palabras Clave: Encía, evaluación, profundidad de surco, hilo retractor. (Fuente: DeCS BIREME)

\begin{abstract}
Objective: To evaluate the depth of the gingival sulcus after the use of retractor thread 00 for class $V$ restorations. Materials and Methods: For the present investigation, 84 dental pieces were evaluated in 46 patients, both men and women who attended the Clinic of the University of the Americas. Probing and control were performed at 72 hours to determine if it affected the retractor thread at the level of the gingival sulcus. Results: $64.29 \%$ of the pieces evaluated showed a change, while $35.71 \%$ remained in the normal standard. Regarding the differentiation of gingival biotypes, a greater change in the measurement of the gingival sulcus was obtained in patients with a thin biotype with $64.81 \%$ of the pieces evaluated in relation to the gross biotype with $35.19 \%$. In the present study, the existence of bleeding at the level of the gingival sulcus was also determined at the time of the drilling: initial, middle and final. With bleeding results of $7.69 \%, 34.62 \%$ and $57.69 \%$ respectively. Conclusion: It was determined that there are significant differences with respect to the depth of the furrow presented in the initial, middle and final drilli ng, however the level of depth of the furrow was higher in the Middle Probe. This confirms that the use of the retractor thread a ffects the periodontium when performing the restorative procedure. In this study it was shown that $64.29 \%$ of the pieces evaluated were affected.
\end{abstract}

Keywords: Gingiva, evaluation, depth of groove, retractor thread. (Source: MeSH NLM)

Recibido: 15 de enero de 2018

Aprobado: 20 de marzo de 2018

Publicado: 30 de marzo de 2018

${ }^{1}$ Universidad de las Américas.

¿Universidad Tecnológica Equinoccial.

${ }^{\mathrm{a} O d o n t o ́ l o g o . ~}$

Correspondencia:

Alexander Cruz Gallegos

Correo electrónico: alexandercruz1993@hotmail.com

Este es un artículo Open Access distribuido bajo la licencia Creative Commons Atribución-NoComercial- Compartir Igual 4.0

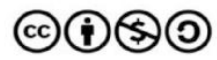

Citar como: Giacometti - Velasco S, Cruz Gallegos A, Quintana - Ramírez A. Evaluación de la profundidad del surco gingival después del uso del hilo retractor 00 para restauraciones clase $v$ en pacientes atendidos en la clínica odontológica de la Universidad de las Américas. KIRU. 2018; 15(1): 36 - 41. https://doi.org/10.24265/kiru.2018.v15n1.04 


\section{INTRODUCCIÓN}

La relación entre la salud periodontal y la restauración de los dientes es íntima e inseparable con el fin de obtener una armonía clínica y estética al paciente. Para que una restauración sea exitosa y perdure por un largo plazo el periodonto debe estar en condiciones saludables ${ }^{(1)}$.

Para que el periodonto no se vea afectado y permanezca saludable la o las restauraciones a este nivel deben realizarse con mucha cautela ya que estéticamente juegan un papel importante, lo mismo que en el equilibrio ecológico de la placa y en el mantenimiento del periodonto. Si una restauración tiene que ser colocada a nivel supragingival, no existe la necesidad de realizar la retracción gingival. Sin embargo, existen ciertas restauraciones (clase $\mathrm{V}$ de Black) a nivel del cuello dental que nos obligan al uso de procedimientos de retracción gingival $\mathrm{y}$, en este caso, al manejo de hilo retractor (con fines estéticos para realizar una restauración óptima al paciente). El mal uso de este puede desencadenar una invasión al espacio biológico y afectar el ancho del mismo. El espacio biológico no debe ser agredido en cualquier procedimiento de restauración (2).

El uso del hilo retractor en cualquier tratamiento odontológico tiende a producir una ligera dilatación a nivel de la encía; esto puede afectar los valores normales del surco gingival en un mal uso, lo que sucede debido a una invasión del espacio biológico y produce cambios en los valores normales del surco provocando ciertos problemas como: retracción gingival, pérdida ósea, hiperplasia gingival. Asimismo, causa un problema tanto a nivel periodontal como estético ${ }^{(3)}$.

El tiempo de exposición del hilo también es un factor que contribuye a desencadenar los problemas mencionados; es por eso que controlar el tiempo requerido para que el hilo permanezca en boca es indispensable. Además, los hilos de retracción gingival pueden contener químicos que favorecen la hemostasia pero que pueden afectar y alterar los valores saludables del surco gingival. La manipulación por parte del odontólogo es esencial para no dañar los tejidos y causar dolor o malestar al paciente (4)

La importancia del espacio biológico es indispensable ya que una alteración a este nivel puede incitar a problemas como retracción gingival, hiperplasia gingival, pérdida ósea, entre otros. Es por eso que el espacio biológico no debe ser agredido bajo ningún procedimiento restaurador (5).
Las dimensiones del espacio biológico aproximadamente son de $1,07 \mathrm{~mm}$ de tejido concetivo, que se encuentra por encima de la cresta alveolar y del epitelio de unión, y, en la parte inferior del surco gingival, el espacio por encima del tejido concetivo ocupa $0,97 \mathrm{~mm}$. La suma de estos dos valores constituye el espacio biológico ${ }^{(6)}$.

En cuanto al aspecto histológico, se afirma que el tejido conectivo está constituido por fibroblastos en un $5 \%$, células de los vasos sanguíneos, linfáticos y terminaciones nerviosos en un $35 \%$ y fibras colágenas de tipo 1 en un $60 \%$. El epitelio de unión se define como un epitelio no queratinizado escamoso y estratificado, su organización celular está dada por queranocitos y no queranocitos $O$ células raras (linfocitos $T$ y $B$, macrófagos, melanocitos) ${ }^{(5)}$.

La evaluación del espacio biológico se puede calcular con la ayuda de la sonda periodontal. Se inicia sondeando desde la cresta alveolar y a este valor se le resta el valor de la profundidad del surco. Este procedimiento se realiza bajo anestesia y ayuda a identificar los valores normales del espacio biológico y así diagnosticar si presenta una invasión a este nivel ${ }^{(7)}$.

Las medidas obtenidas por el sondaje periodontal son bastante certeras, pero en ciertos casos se pueden presentar errores debido a la fuerza sobre la sonda, el diseño de la misma, el paciente, el examinador, la profundidad de la bolsa, inflamación del tejido y ubicación de las fibras dentogingivales. Entre algunas de las sondas más utilizadas tenemos: Marquis (193097-Hu Friedy ${ }^{\circledR}$ Mfg.Co.), OMS (500593-Hu Friedy®Mfg. Co.), Goldman Fox (Premiun Plus-Pakistan) y Universidad Carolina del Norte (UCN) (937711- Hu Friedy 8 Mfg. Co), entre otras ${ }^{(8)}$. El biotipo periodontal juega un papel importante en la sonrisa de cada paciente, que está relacionada con el tamaño de los labios, la morfología y tamaño de los dientes y el aspecto gingival que se logre visualizar. Es por eso que la interacción a nivel del margen gingival debe ser con cautela, ya que un error a este nivel puede provocar un cambio a nivel de la encía y comprometer la estética del paciente ${ }^{(9)}$

El método mecánico consiste en desplazar físicamente los tejidos mediante la colocación de una materia entre la preparación y el tejido gingival. El más usado es el hilo retractor. En este método son empleadas bandas de cobre o aluminio las cuales ayudan a una buena adaptación del margen sin dañar tejidos blandos. En la actualidad se utilizan siliconas para sustituir a la modelina y cofias de acrílico a las bandas de cobre. 
Este es un método traumático, por lo que la técnica del operador deberá ser de mucho conocimiento ya que se puede lesionar con facilidad al epitelio de unión ${ }^{(10)}$.

En cuanto al procedimiento, primeramente se aísla el diente con rollos de algodón y el campo operatorio debe estar seco. Se corta la longitud con que se desea rodear al diente. Se aconseja sumergir en una solución astringente para evitar que el epitelio del surco se adhiera a este y cause desgarro al momento de retirarlo.

El siguiente paso es rodear el diente con el hilo y proceder a introducirlo dentro del surco con un instrumento adecuado. Se aconseja empezar desde proximal y seguir por la parte lingual o palatina. Se recomienda que el empaquetamiento del hilo sea suave pero con firmeza. Se debe evitar compactar con fuerza excesiva ya que puede causar un desgarro en la inserción gingival y evitar el uso del hilo repetidas veces en un mismo diente ya que puede desencadenar retracción gingival ${ }^{(11)}$.

\section{MATERIALES Y MÉTODOS}

El universo de la presente investigación estuvo constituido por los pacientes que acuden a la Clínica de Odontología de la Universidad de las Américas, ubicada en el municipio de Quito, Ecuador. Fueron seleccionados 46 pacientes y fueron evaluados 84 dientes según los criterios de inclusión y exclusión.

En cuanto al análisis estadístico se aplicó una prueba de Shapiro - Wilks (modificado) y se realizó una gráfica de Q-Q plot para demostrar la normalidad de los datos. Ya que no hubo normalidad en los datos se prosiguió a la aplicación de la prueba de Kruskal Wallis para la obtención normal de los mismos.

El tipo de instrumento utilizado para este estudio fue la observación clínica, ya que con las medidas obtenidas por el sondaje del suco gingival al momento de colocar el hilo retractor se recolectaron los hallazgos obtenidos.

Criterios de Inclusión: Pacientes de sexo masculino y femenino que acuden a la Clínica Odontológica de la Universidad de las Américas. Pacientes con lesiones clase $\mathrm{V}$ no cariosas en los premolares y caninos superiores o inferiores. Pacientes con buena salud periodontal. Índice de placa menor al $50 \%$.

Criterios de Exclusión: Enfermedad periodontal (gingivitis o periodontitis) y que la colocación del hilo no esté indicada. Pacientes fumadores o diabéticos. Pacientes en estado de gestación. Lesiones clase $\mathrm{V}$ de tipo carioso. Enfermedades sistémicas. Menores de 18 años.

Las personas que forman parte de nuestro estudio fueron evaluadas por un examen clínico previo para identificar si tenían algún tipo de problema bucal. Se realizó la apertura de la historia clínica y se evaluó el índice de placa, índice de caries y estado periodontal. Para la valoración periodontal se realizó el Periodontal Screening Recording (PSR) utilizando la sonda periodontal de la Universidad de Carolina del Norte. Asimismo, se realizó el sondaje por sextantes, un diente por cada uno de ellos. Posteriormente se realizó la profilaxis para obtener un ambiente limpio antes de cualquier tratamiento.

El hilo retractor empleado para este estudio (Hilo retractor 00) es de tipo trenzado y no posee agentes químicos incorporados. En cuanto al procedimiento y técnica del hilo retractor, primero se aísla el campo con torundas de algodón, donde es colocado el hilo retractor y se selecciona y corta el largo deseado para que pueda circundar la preparación. Seguidamente, se determina el espesor deseado de acuerdo con el ancho y profundidad del surco.

La colocación del hilo se realizó en un campo seco con un leve chorro de aire para ayudar a una mejor inserción dentro del surco. Con la ayuda del empaquetador de hilo, se rodea al diente con el hilo y se introduce suavemente dentro del surco, lo que se inicia por la zona interproximal ya que es más fácil su ingreso, y se continúa por la zona palatina o lingual con una inclinación de $45^{\circ}$ presionando el hilo contra el diente. Para evitar el desalojo por la tensión del material de expansión gingival, se sugiere que el instrumento de empaquetado se presione en dirección contraria a la dirección del empaquetado. El tiempo para obtener dilatación de la encía es de 3 a 5 minutos.

El factor del tiempo es determinante al momento de la colocación del hilo retractor. Lo aconsejable es dejar que el hilo actué a nivel del surco alrededor de 3 a 4 minutos para q produzca la dilatación optima para el procedimiento restaurador $^{(12)}$.

\section{RESULTADOS}

Se obtuvieron los objetivos planteados, los mismos que están representados por el género de los pacientes, el cambio o no de las medidas del surco gingival, el biotipo periodontal más afectado y la presencia $o$ no de sangrado. 
Se evaluó la profundidad del surco gingival después de colocar el hilo retractor 00 para restauraciones clase $\mathrm{V}$ en un total de 46 pacientes atendidos en la clínica de la Universidad de las Américas. Se atendió un total de 22 pacientes de sexo masculino y 24 pacientes de sexo femenino, lo que representa el $47,83 \%$ y el $52,17 \%$, respectivamente.

Se colocó el hilo retractor en un total de 84 piezas dentales pertenecientes a los 46 pacientes evaluados, donde se determinó que 46 piezas poseían un biotipo gingival delgado, mientras que 38 piezas presentaron un biotipo grueso, lo que corresponde al 54,76 \% y 45,24\%, respectivamente.

Se midió el nivel de profundidad del surco después del uso del hilo retractor. Con el fin de reducir el error estándar, se realizaron tres mediciones por cada pieza dental evaluada: sondaje inicial, medio (después de retirar el hilo) y final (después de 3 días del procedimiento). Se observó que existió una mayor profundidad del surco en el caso del sondaje medio que presentó una media de $1,73 \mathrm{~mm}$, en tanto que el sondaje inicial presentó una media de $1,23 \mathrm{~mm}$ y el sondaje final, $1,38 \mathrm{~mm}$. Cabe aclarar que la medida media se realizó inmediatamente después de retirar el hilo retractor, la cual nos da un valor relativamente mayor a los de las medidas inicial y final. El epitelio presentaba el estimulo del hilo retractor, el cual se vio claramente reflejado en la medida media.

Se aplicó una prueba de Shapiro - Wilks (modificado) y se realizó una gráfica de $\mathrm{Q}-\mathrm{Q}$ plot para determinar si los datos tienen una distribución normal. En la Tabla 3 se observa la prueba de Shapiro - Wilks (modificado), donde el valor de $p$ fue $<0,0001(p=0,01 \%)$, el cual es menor al nivel de confianza de la prueba ( $\alpha=5 \%$ ), lo que nos indica que los datos no están distribuidos normalmente, tal y como se observa en la gráfica de $Q-Q$ plot, por lo que los datos proporcionados por el ANOVA y la prueba de Duncan no se aceptan.

Tabla 1. Prueba de Shapiro - Wilks (modificado) para la comparación de medias respecto a los niveles de profundidad del surco para los sondajes inicial, medio y final.

Shapiro-Wilks (modificado)
\begin{tabular}{clrlll} 
Variable & n & Media & D.E. & W* & (Unilateral D) \\
\hline RDUO Retracción & 756 & 0,00 & 0,64 & 0,93 & $<0,0001$ \\
\hline
\end{tabular}

Por lo tanto, clasifica al nivel de profundidad del surco del sondaje inicial en el subgrupo $A$, al del sondaje final en el subgrupo B y, finalmente, al del sondaje medio en el subgrupo $C$, siendo este el sondaje en el que se obtuvo el mayor nivel de profundidad del surco, pues obtuvo la media más alta de los tres sondajes $(1,73$ $\mathrm{mm})$.

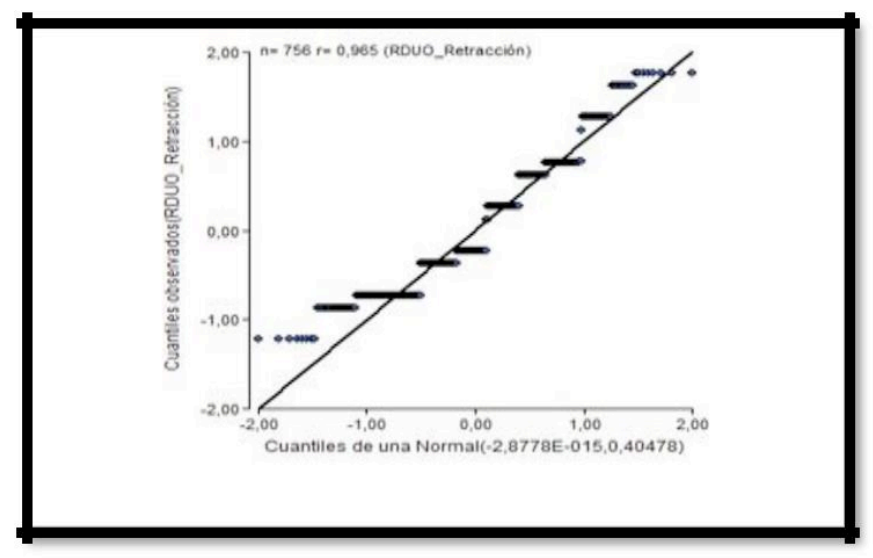

Tabla 2. Prueba de Kruskal Wallis con respecto a los niveles de profundidad del surco para los sondajes inicial, medio y final.

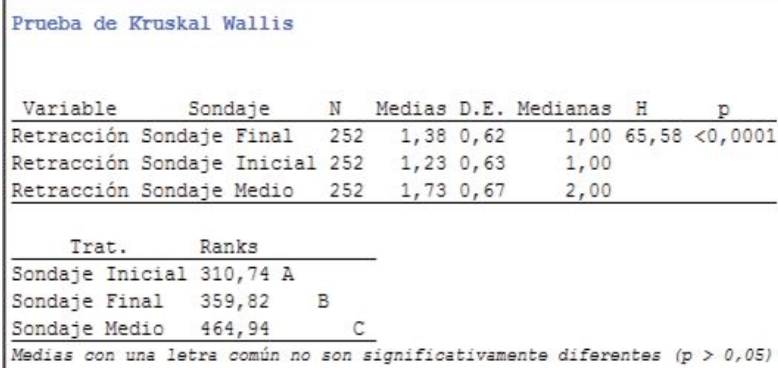

Posteriormente, se analizó los niveles de profundidad del surco en el sondaje inicial, medio y final. Se asignó el valor de 1 a las piezas que presentaron cambio y el valor de 0 a las que no.

De las 84 piezas evaluadas, se determinó que 54 piezas efectivamente presentaron un cambio con respecto al nivel de profundidad del surco después de la colocación del hilo retractor 00 , mientras que 30 piezas no presentaron ningún cambio, lo cual representa un $64,29 \%$ de piezas que cambiaron frente al $35,71 \%$ de aquellas que no presentaron cambios en la medida del surco gingival. 
Dentro de las 54 piezas dentales que cambiaron la medida del surco gingival después de la colocación del hilo retractor 00 , se determinó que 35 poseían un biotipo delgado, mientras que 19 tenían un biotipo gingival grueso, lo que corresponde al $64,81 \%$ y al $35,19 \%$ respectivamente, usando como parámetro para los dos valores la medida media.

Tomando en cuenta que los datos correspondientes al cambio en la medida del surco gingival según el biotipo de piezas evaluadas no cumplen con la normalidad, se realizó un análisis de varianza no paramétrico, por lo que se aplicó la prueba de Kruskal Wallis, en la que se obtuvo un valor de $p=0,0136(p=1,36 \%)$, menor al nivel de significancia del $5 \%$, lo que indica que hay diferencias significativas entre las piezas que presentaron un cambio en la medida del surco gingival según el biotipo de las mismas. Se agrupó a las piezas que poseía un biotipo gingival grueso en el subgrupo A, mientras que las piezas con un biotipo gingival delgado fueron agrupadas en el subgrupo $B$, lo que nos indica que existe diferencia estadística significativa con respecto al cambio en la medida del surco gingival de las piezas con un biotipo grueso y delgado. En el caso de las piezas con biotipo delgado, hubo una mayor cantidad de piezas que cambiaron ya que sus medias tuvieron un valor superior $(0,76)$, tal como se muestra en la Tabla 3.

Tabla 3. Prueba de Shapiro - Wilks (modificado) para el cambio en la medida del surco gingival según el biotipo de piezas evaluadas.

\begin{tabular}{|l}
\hline Shapiro-Wilks (modificado) \\
\begin{tabular}{llllll|} 
Variable & n & Media & D.E. & $W^{*}$ & $p$ (Unilateral D) \\
\hline RDUO Cambio & 84 & 0,00 & 0,46 & 0,76 & $<0,0001$ \\
\hline
\end{tabular} \\
\hline
\end{tabular}

Se evaluó también los diferentes tipos de sangrado que se presentaron en las diferentes piezas dentales. Se determinó que 2 piezas presentaron sangrado inicial, lo que equivale al 7,69\%. 15 piezas dentales presentaron sangrado final, que corresponde al 57,69\%, mientras que 9 piezas presentaron sangrado medio, que es igual al $34,62 \%$.

\section{DISCUSIÓN}

El objetivo del presente estudio fue evaluar la profundidad del surco gingival después del uso del hilo retractor 00 para restauraciones clase $V$, en tratamientos restauradores en la clínica de la Universidad de las Américas. En los resultados se demostró una invasión del surco en el $64,29 \%$ de los dientes tratados y el $35,71 \%$ de dientes que no tuvieron ningún cambio. Estos resultados confirman la investigación realizada por Nugala et al. ${ }^{(13)}$ que resaltan la intima relación que tiene el periodonto con los tratamientos odontológicos restauradores.

Hay que tener en cuenta que las mediciones, tanto inicial como media, se realizaron inmediatamente después de retirar el hilo retractor, dando como medidas $1,23 \mathrm{~mm}$ y $1,73 \mathrm{~mm}$, respectivamente, y que la medida final se hizo tres días después del tratamiento, la cual disminuyó notablemente al compararla con la medida media y la inicial, ya que el este tejido no presentaba el estimulo del hilo retractor. En consecuencia, no hay una diferencia significativa entre la medida inicial y la final.

Otro estudio realizado por Santos et al. ${ }^{(14)}$ afirma que el procedimiento restaurador tiene íntima relación con el tejido periodontal; es por eso que se debe evaluar el espacio biológico antes y después del procedimiento restaurador para determinar si existió una invasión a este nivel.

Se demostró en otros estudios la medida del espacio biológico que osciló entre 2,73 $\mathrm{mm}$ y $3,23 \mathrm{~mm}$, respectivamente. Para obtener resultados más precisos realizaron cortes histológicos del espacio biológico ${ }^{(5)}$.

Para realizar un diagnóstico que nos indique si hay una invasión o compromiso del espacio biológico en un diente, se debe realizar un sondaje para obtener valores que nos certifiquen que hubo dicha invasión. En el estudio de Rodriguez et al. ${ }^{(15)}$, los valores de la medición del espacio biológico aproximadamente son de $3 \mathrm{~mm}$; específicamente respecto al surco gingival presenta un valor que oscila entre $0,69 \mathrm{~mm}$ y $1,32 \mathrm{~mm}$. La investigación presentó una medida media de todas las piezas estudiadas, obteniendo un valor de 1,22 mm en el momento de diagnóstico periodontal.

A nivel del surco gingival, Delgado et al. demostraron que en dientes restaurados se obtenía una medida de entre 1,60 y $0,80 \mathrm{~mm}$, y en no restaurados de $1,27-0,79 \mathrm{~mm}$, en tanto que las medidas de profundidad de surco entran en los rangos para dientes restaurados con un valor de 1,37

$\mathrm{mm}$ y antes del procedimiento restaurador, con un valor de $1,22 \mathrm{~mm}$.

\section{CONCLUSIÓN}

Tomando en consideración los resultados arrojados por el análisis estadístico, se determinó que existen diferencias significativas en la profundidad del surco 
presentada en los sondajes inicial, medio y final; sin embargo el nivel de profundidad del surco fue superior en el sondaje medio,

o que confirma que el uso del hilo retractor afecta al periodonto en el momento de realizar el procedimiento restaurador. Se demostró una invasión del surco en $64,29 \%$ de los dientes tratados y un $35,71 \%$ de dientes que no tuvieron ningún cambio. Estos resultados resaltan la íntima relación que tiene el periodonto con los tratamientos odontológicos restauradores.

Se obtuvo como resultado que el mal uso del hilo retractor produce sangrado a nivel del surco gingival. El estudio determinó un mayor porcentaje de sangrado en $57,69 \%$ de piezas evaluadas, por lo que se concluye que existe una invasión del surco gingival.

Contribuciones de autoría: SGV, ACG, AQR. Participó en la concepción o diseño del estudio, revisión de la literatura, participó en el aporte de material de estudio, recolección/ obtención de los datos, análisis e interpretación de resultados. Brindó asesoría estadística. Redacción del artículo. Revisión crítica del artículo. Aprobación de la versión final del artículo.

Fuente de financiamiento: Autofinanciado.

Conflicto de intereses: No existe un posible conflicto de intereses en este manuscrito. Si existiera, será declarado en este documento y/o explicado en la página del título.

\section{REFERENCIAS BIBLIOGRÁFICAS}

1. Matta E, Alarcon M. Matta C. Espacio biológico y prótesis fija: Del concepto clásico a la aplicación tecnológica. Revista Estomatologica Herediana. 2012; 22(2).

2. Phatale S, Marawar PP, Byakod G, Lagdive SB, Kalburge JV. Effect of retraction material son gingival health: A histopathological study. Journal of Indian Society of Periodontology. 2010; 14: 35-39.

3. Baratieri $L$ et al. Odontologia restauradora: fundamentos y técnica, volumen 1. Sao Paulo: Santos, 2011.

4. Santos P. Por qué y cómo utilizar la cofia en la toma de impresión en prótesis parcial fija. Revista Odontológica Dominicana. 1993; № 2.

5. Delgado Pichel A, Inarejos Montesinos P, Herrero Climent M. Espacio biológico. Parte I: La inserción diente-encía. Av Periodon Implantol. 2001; 13,2: 101-108.

6. Carranza F, NewmanM, Takei H. Carranza's clinical periodontology-9th ed. Philadelphia: W.B. Saunders Co; 2002.

7. Maheaswari R, GollaUsha R, Logarani A, Sudagaram M, Rohan B. Biologic Width - Critical Zone for a Healthy Restoration. IOSR Journal of Dental and Medical Sciences (IOSR-JDM). 2014; 13(2); 93-98.
8. Adrianzén $\mathrm{C}, \mathrm{Coz} \mathrm{M}$, Noriega J. Evaluación del sondaje in vitro con cuatro sondas periodontales manuales, considerando el factor experiencia del examinador. Revista Estomatológica Herediana. 2010; 20(3): 119-126.

9. Zerón A. Biotipos, fenotipos y genotipos. ¿Qué biotipo tenemos? (Segunda parte). Revista Mexicana de Periodontología.2011; 2(1): 22-33.

10. Salazar, J. Métodos de separación gingival en prótesis fija. Acta Odontológica Venezolana. 2007; 45(2).

11. Reyes F, Mosqueda R. Consideraciones ideales en la toma de impresión dental. Revista ADM. 2001; LVIII(5):183-190.

12. Gupta A, Prithviraj D, Gupta D, Shruti DP. Clinical Evaluation of Three New Gingival Retraction Systems: A Research Report. J Indian Prosthodont Soc. 2013; 13(1): 36-42.

13. Nugala, B, Kumar, S, Sahitya, S, Krishna. Biologic width and its importance in periodontal and restorative dentistry. Journal of Conservative Dentistry. 2012; 15(1): 12-17.

14. Santos E, Lima R, Rocha C. Fractura dental con invasión del espacio biológico - una conducta multidisciplinar. Acta Odontol Venezolana. 2010; 48(1).

15. Rodriguez, A, Bravo, F, Pomarino S. Manejo del espacio biológico - revisión de literatura. Acta Odontol Venezolana. 2014: 52(3). 